\title{
Preoperative Uric Acid-to-albumin Ratio as a New Indicator for Predicting Long-term Prognosis in Patients With Acute Type a Aortic Dissection
}

\section{Xue Wang}

First Affiliated Hospital of Xi'an Jiaotong University

\section{Chao Deng}

First Affiliated Hospital of Xi'an Jiaotong University

Fengwei Guo

First Affiliated Hospital of Xi'an Jiaotong University

\section{Liang Zhong}

First Affiliated Hospital of Xi'an Jiaotong University

\section{Ming Li}

First Affiliated Hospital of Xi'an Jiaotong University

\section{Yanbo Xue}

First Affiliated Hospital of Xi'an Jiaotong University

\section{Qiong Ma}

First Affiliated Hospital of Xi'an Jiaotong University

\section{Xiaopu Zheng}

First Affiliated Hospital of Xi'an Jiaotong University

Heng Gao ( $\nabla$ gaoheng19820109@163.com )

Shaanxi Provincial People's Hospital

\section{Research Article}

Keywords: acute aortic dissectionn, uric acid-to-albumin Ratio, serum albumin

Posted Date: January 27th, 2022

DOI: https://doi.org/10.21203/rs.3.rs-1281513/v1

License: (c) (1) This work is licensed under a Creative Commons Attribution 4.0 International License. Read Full License 


\section{Abstract}

Objectives - We aimed to analyze the ability of the preoperative uric acid-to-albumin ratio (UAR) to predict the postoperative long-term mortality of patients with type A AAD.

Methods -A total of 289 patients with type A AAD who were enrolled in this study. First, all patients were divided into two groups: UAR $<9.875$ and UAR $\geq 9.875$. Furthermore, according to whether death occurred after one year after surgery, the patients were divided into the non-survival and survival groups. Univariable and multivariable COX regression analyses were conducted to identify the independent risk factors for long-term death in patients with type A AAD. Receiver operating characteristic (ROC) curves were constructed to assess the values of these indices in predicting postoperative mortality.

Results- Age, hypertension, albumin content, UAR, and D-dimer content were found to be independent risk factors for mortality. The area under the ROC curve (AUC) of UAR was $0.618(\mathrm{HR} 1.904,95 \% \mathrm{Cl}, 1.097$ to 3.305; $P<0.05)$, the combination of UAR, age and $D$-dimer was best for predicting deaths, the area under the AUC of them was 0.751 [HR $1.904,95 \% \mathrm{Cl}, 0.681$ to $0.821 ; \mathrm{P}<0.05)]$.

Conclusions - In patients with type A AAD, UAR may considered as a new independent risk factor for longterm mortality. a combination of UAR, age, and D-dimer content was a best predictor of deaths.

\section{Full Text}

Type A acute aortic dissection (AAD) is the most common and catastrophic presentation of acute aortic syndrome. Most type A AAD patients have severe and unbearable chest pain and rapidly developing complications including aortic dissection rupture, pericardial effusion and tamponade, and acute myocardial infarction. Despite aggressive surgical techniques, the prognosis of some patients remains poor, and the mortality can be as high as $30 \%$. Moreover, it is difficult to identify patients at high risk of death, which is important for rapid decision making [1-3].

Serum albumin (SA) is the most important protein in human serum and has many important physiological functions. The catabolism of SA increases after the activation of inflammatory response. A low SA level can lead to enhanced platelet activation and aggregation along with increased blood viscosity, leading to the damage of endothelial cells [4]. Ronit studied 2240 individuals without cardiovascular diseases in Japan and found that low SA was linked to the occurrence of hypertension [5]. Furthermore, low SA levels can lead to pulmonary edema and fluid retention, which aggravate primary cardiovascular disease and are independent predictors of ischemic heart disease [6-8].

Elevated serum uric acid concentrations can increase the expressions of chemokines and cytokines in the vascular system, activate the renin angiotensin system, and increase the expression of c-reactive protein, resulting in a cascade reaction of inflammation and oxidative stress and damage to vascular endothelial cells $[9,10]$. Uric acid has been shown to be an independent factor in the occurrence and development of cardiovascular events such as ascending aortic dilatation, hypertension, coronary heart disease, 
cerebrovascular disease and other cardiovascular diseases [11-13].Çakmak reported that the uric acid-toalbumin ratio (UAR) is a novel inflammatory marker that independently provides better predictive ability for outcomes than the C-reactive protein-to-albumin ratio; thus, UAR might be useful for predicting the extent of in on-ST-elevation myocardial infarction囚NSTEMI $₫$

patients [14]. However, no studies have been conducted on the effect of UAR on the prognosis of patients with type A AAD, and whether the predictive value of UAR for prognosis differs from SA level or uric acid level. Therefore, we investigated the connection between preoperative UAR and the prognosis of patients with type A AAD. Moreover, the evaluated the predictive value of UAR by observing follow-up patients with type A AAD.

\section{Patients and Methods}

All patients in this study were patients at the Cardiovascular Surgery Department of the First Affiliated Hospital of Xi'an Jiaotong University from January 2019 to September 2020. These patients were diagnosed with type A AAD by computed tomography. Dissection was classified according to the Stanford criteria. The primary inclusion criteria were patients with Stanford type A AAD within two weeks after symptom onset and aged 18 to 75 years. The exclusion criteria were as follows: (1) a history of cardiogenic shock or pericardial tamponade; (2) iatrogenic aortic dissection; (3) traumatic aortic dissection; (4) severe valve disease; (5) congenital heart disease; (6) severe organ dysfunction such as liver or kidney failure; (7) metabolic diseases; (8) malignant tumors; (9) gout or thyroid disease; (10) severe gastrointestinal diseases; and (11) recent use of drugs affecting uric acid and albumin. A total of 289 patients with type A AAD were admitted to our study. Patients were followed-up between September and October 2021. The primary endpoint event was death, and the secondary endpoint event was a second surgery. The clinical data of the enrolled patients were obtained by consulting their medical records. Demographic information, medical history, vital signs, and laboratory test results were also recorded. Complications and outcomes were recorded during the follow-up period. All data were analyzed in a blind manner.This study was approved by the Research Ethics Committee of the First Afliated Hospital of Xi'an Jiaotong University and the written informed consent was given by all subjects. All the study protocols were followed in the guidelines of the Research Committee of Human Investigation of Xi'an Jiaotong University Health Science Center.

\section{Surgical method}

All patients with type A AAD were surgically treated within $1 \mathrm{~d}$ after admission. A stent-graft (MicroPort Medical Company Limited, Shanghai, China) and four-branched prosthetic graft (Vascutek Limited 4 Branch Graf, Newmains Avenue, Inchinnan, Renfrewshire, Terumo) were used in total arch replacement combining with the implantation of a stented elephant trunk. Patients underwent a median sternotomy and total cardiopulmonary bypass.

\section{Methods for detecting clinical indicators}


Peripheral blood samples were collected before anesthetic induction.Clinical indicators [platelet count, leukocyte count, red blood cell count, hemoglobin, and C-reactive protein囚CRP囚]were evaluated using an automatic blood cell analyzer (Mindray, Model: BC6800Plus, Shenzhen Mindray Bio-Medical Electronics Co., Ltd., China). CRP was detected using an automatic blood cell analyzer (Mindray, Model: CRP-M100, Shenzhen Mindray Bio-Medical Electronics Co., Ltd., China). Total bilirubin, albumin, globulin, total cholesterol, urea, creatinine, uric acid, and creatine kinase-MB $₫ C K-M B \bigotimes$ contents were detected using an automatic biochemical analyzer (HITACHI, Model: 008AS, Japan). The D-dimer and fibrinogen degradation products (FDP) contents were detected using a hemagglutination analyser-3 instrument (SYSMEX, Model: Cs-5100, Japan), high-sensitivity troponin were detected using automatic analyzer (RADIOMETER, model ABL90FLEX, Radomite Medical Equipment Co., LTD.)

\section{Statistical Analysis}

Summary statistics are presented as frequencies and percentages or as means \pm standard deviation (SD). Differences between two groups were compared using unpaired Student's t tests or Mann-Whitney $\mathrm{U}$ tests for continuous variables and $\chi^{2}$ tests for categorical variables. The COX proportional hazards model was used to identify predominant predictors for aortic events throughout the entire follow-up period based on univariate and stepwise multivariate analyses. Survival rate, dissection-related death-free rate, and aortic event-free rate were computed according to the Kaplan-Meier technique, and event-free curves were compared by log-rank test. For laboratory results, we also assessed whether measurements were outside the normal range. All statistical analyses were performed using IBM Statistics 26. A twotailed value of $P<0.05$ was considered statistically significant.

Consent for publication. Our study does not contain any individual person's data in any form. All authors have signed a consent form for publication in case of acceptance.

\section{Results}

\section{Basic and clinical characteristics}

A total of 289 patients were included in this study. Each patient received a surgical operation. Between January 2019 and September 2020, 213 men and 76 women were enrolled with an average age of $52 \pm$ 11 years. All patients were divided into the UAR $<9.875$ group (135 patients) and the UAR $\geq 9.875$ group (154 patients) according to the optimal critical value of UAR in ROC analysis. The indicators of age, men, BMI, globulin, total bilirubin, BUN, Cr, creatinine clearance, HGB, WBC, RBC, total cholesterol, CK-MB, hypersensitive troponin $\mathrm{T}$, ventilator-assisted time, length of stay in the ICU, and mortality) were significantly different between the two groups $₫ T$ Table $1 \rrbracket$. According to whether death occurred after the procedure during follow-up, the patients were further divided into the death and survival groups. The indicators of age, hypertension, albumin, UAR, Cr, creatinine, clearance, RB, CPLT, SBP, CK-MB, D-dimer, and FDP were significantly different between the two groups $₫$ Table 2区. As shown in Figure 1, KaplanMeier survival analysis indicated that mortality in patients with type A AAD was higher in patients with high UAR $(\geq 9.875 \mu \mathrm{mol} / \mathrm{g})$ compared to those with UAR $\left(<9.875 \mu \mathrm{mol} / \mathrm{g}\right.$; log-rank $\left.x^{2}=20.68 ; P<0.001\right)$. 
Table 1. Baseline and clinical characteristics of the UAR $\geq 9.875$ and UAR $<9.875$ groups. Data are mean $\pm \mathrm{SD}$ or median (interquartile range), $n(\%)$. BMl, body mass index; $\mathrm{CHD}$, coronary heart disease; $\mathrm{BUN}$, urea nitrogen; $\mathrm{Cr}$, creatinine; $\mathrm{HGB}$, hemoglobin; $\mathrm{HGB}$, hemoglobin; WBC, white blood cells;RBC, red blood cells; PLT: platelet; CRP, C reactive protein; SBP, systolic blood pressure; $\mathrm{DBP}$, diastolic blood pressure; $\mathrm{HR}$, heart rate; $\mathrm{CK}-\mathrm{MB}$, creatine kinade $\mathrm{MB}$; $\mathrm{ICU}$, intensive care unit.

Table 2. Baseline and clinical characteristics of the death and survival groups. Data are mean \pm SD or median (interquartile range), $n(\%)$. FDP, fibrinogen degradation product.

\section{Predicting factors for death}

COX regression multivariate analysis after univariate analysis of all-cause mortality indicated the following independent risk factors for mortality (Table 3): UAR (hazard ratio [HR], 1.904; 95\% confidence interval [Cl], 1.097 to $3.305 ; P<0.05)$, age (HR, 2.216; $95 \% \mathrm{Cl}, 1.287$ to $3.815 ; P<$ 0.05), hypertension (HR, 1.825; $95 \% \mathrm{Cl}, 1.077$ to $3.094 ; P<0.05)$, and $\mathrm{D}$-dimer ( $\mathrm{HR}, 1.991 ; 95 \% \mathrm{Cl}, 1.116$ to $3.554 ; p<0.05)$. Patients in the UAR $\geq 9.875$ group had a significantly higher death rate than those in the UAR $<9.875$ group (Figure $1 ; P<0.001$ ). The predictive values of UAR, D-dimer, age, hypertension, albumin, and uric acid for death was evaluated using the receiver operating characteristic (ROC) method, as shown in Table 4.

As shown in Figures 2 and 3, the patients were divided into two groups according to the optimal critical value of UAR: high UAR $(\geq 9.875 \mu \mathrm{mol} / \mathrm{g})$ and low UAR $(<9.875 \mu \mathrm{mol} / \mathrm{g})$. Of the 135 patients in the low UAR group, 21 (13.6\%) died during follow-up. In contrast, of the 154 patients in the high UAR group, 48 (31.2\%) died during follow-up. Chi-square test showed a significant difference in mortality between the two groups $(P=0.002)$. For $\mathrm{D}$-dimer, the patients were divided into two groups according to the optimal critical D-dimer content: high D-dimer $(>9.19 \mathrm{~g} / \mathrm{L})$ and low D-dimer $(\leq 9.19 \mathrm{~g} / \mathrm{L})$. The percentages of patients that died during follow-up were $37.6 \%$ in the high D-dimer group but only $12.2 \%$ in the low $D$ dimer group, and the difference in mortality was significant based on Chi-square test $(P=0.000)$.

For age, the patients were divided into two groups according to the optimal cutoff value: high age ( $>56$ years) and low age ( $\leq 56$ years). In the high age group, $38.7 \%$ of patients died during hospitalization, while only $15.3 \%$ of patients in the low age group died. This difference was significant based on Chisquare test $(P=0.103)$. For albumin, the patients were divided into two groups according to the optimal critical value: high albumin $(\geq 36.45 \mathrm{~g} / \mathrm{L})$ and low albumin $(<36.45 \mathrm{~g} / \mathrm{L})$. In the low albumin group, $31.3 \%$ of patients died during follow-up, while the percentage was $14.3 \%$ in the high albumin group. This difference was significant based on Chi-square test $(P=0.001)$. For uric acid, the patients were divided into two groups according to the optimal critical value: high uric acid ( $\geq 305 \mu \mathrm{mol} / \mathrm{L}$ ) and low uric acid (< $305 \mu \mathrm{mol} / \mathrm{L}$ ). In the low uric acid group, $16.0 \%$ of patients died during hospitalization, while $27.7 \%$ of patients in the high uric acid died. This difference was significant based on Chi-square test $(P=0.028)$. For hypertension, the patients were divided into two groups: hypertension and non-hypertension. During follow-up, $16.8 \%$ of patients in the non-hypertension group died, compared to $28.8 \%$ in the hypertension group. This difference was significant based on Chi-square test $(P=0.018)$. 


\begin{tabular}{|c|c|c|c|c|}
\hline Variable & All $(n=289)$ & $\begin{array}{l}\text { UAR }<9.875 \\
\text { group } \\
(n=135)\end{array}$ & $\begin{array}{l}\text { UAR } \geq 9.875 \\
\text { group } \\
(n=154)\end{array}$ & $P$-value \\
\hline Age (years) & $52(45-59)$ & $54(47-61 \rrbracket$ & $51(42-57 \rrbracket$ & $\begin{array}{l}P= \\
0.012\end{array}$ \\
\hline Men, $n(\%)$ & $213(74)$ & $81(600$ & $132(86 \rrbracket$ & $\begin{array}{l}P= \\
0.000\end{array}$ \\
\hline $\mathrm{BMI}\left(\mathrm{kg} / \mathrm{m}^{2}\right)$ & $\begin{array}{l}25.8(22.86- \\
28.15)\end{array}$ & $\begin{array}{l}24.80(22.22- \\
26.24 \rrbracket\end{array}$ & $\begin{array}{l}26.24(24.08- \\
29.33)\end{array}$ & $\begin{array}{l}P= \\
0.002\end{array}$ \\
\hline $\begin{array}{l}\text { Smoking index } \\
\text { (per year) }\end{array}$ & $0(0-400)$ & $0(0-200)$ & $80(0-400)$ & $\begin{array}{l}P= \\
0.004\end{array}$ \\
\hline History of CHD (\%) & $11(4)$ & $4 \otimes 3)$ & $7 \otimes 5)$ & $\begin{array}{l}P= \\
0.483\end{array}$ \\
\hline Hypertension, $n(\%)$ & $170(59)$ & $81(60)$ & $89(58)$ & $\begin{array}{l}P= \\
0.704\end{array}$ \\
\hline Diabetes, n (\%) & $5(2)$ & $2(1)$ & $3(2)$ & $\begin{array}{l}P= \\
0.762\end{array}$ \\
\hline Euroscore $\otimes(\%)$ & $\begin{array}{l}4.65(3.77- \\
5.64)\end{array}$ & $4.65(3.77-5.62)$ & $\begin{array}{l}4.38(3.77- \\
6.16)\end{array}$ & $\begin{array}{l}P= \\
0.668\end{array}$ \\
\hline Globulin (g/L) & $\begin{array}{l}20.5(17.2- \\
24.1)\end{array}$ & $19.1(16.6-21.7)$ & $\begin{array}{l}22.2(17.9- \\
25.3)\end{array}$ & $\begin{array}{l}P= \\
0.000\end{array}$ \\
\hline Total bilirubin $(\mu \mathrm{mol} / \mathrm{L})$ & $\begin{array}{l}30.0(18.1- \\
47.4)\end{array}$ & $38.5(25.9-36.1)$ & $\begin{array}{l}21.1(15.3- \\
35.2)\end{array}$ & $\begin{array}{l}P= \\
0.000\end{array}$ \\
\hline BUN (mmol/L) & $\begin{array}{l}7.13(5.64- \\
9.04)\end{array}$ & $6.61(5.11-8.44)$ & $\begin{array}{l}7.61(5.93- \\
9.99)\end{array}$ & $\begin{array}{l}P= \\
0.000\end{array}$ \\
\hline $\mathrm{Cr}(\mu \mathrm{mol} / \mathrm{L})$ & $78(58-105)$ & $60(44-82)$ & $95(71-125)$ & $\begin{array}{l}P= \\
0.000\end{array}$ \\
\hline $\begin{array}{l}\text { Creatinine clearance } \\
(\mathrm{mL} / \mathrm{min})\end{array}$ & $\begin{array}{l}99.40(69.85- \\
134.25)\end{array}$ & $\begin{array}{l}111.50(79.70- \\
151.70)\end{array}$ & $\begin{array}{l}86.10(57.90- \\
114.70)\end{array}$ & $\begin{array}{l}P= \\
0.000\end{array}$ \\
\hline $\mathrm{HGB}(\mathrm{g} / \mathrm{L})$ & $128 \pm 22$ & $123(112-139)$ & $133(119-146)$ & $\begin{array}{l}P= \\
0.002\end{array}$ \\
\hline WBC $\left(\times 10^{9} / \mathrm{L}\right)$ & $\begin{array}{l}10.76 \\
(8.32+14.43)\end{array}$ & $9.73(7.56-12.72)$ & $\begin{array}{l}12.18(9.48- \\
15.28)\end{array}$ & $\begin{array}{l}P= \\
0.000\end{array}$ \\
\hline $\operatorname{RBC}\left(\times 10^{12} / \mathrm{L}\right)$ & $4.17 \pm 0.63$ & $4.07 \pm 0.52$ & $4.26 \pm 0.90$ & $\begin{array}{l}P= \\
0.003\end{array}$ \\
\hline PLT $\left(\times 10^{9} / \mathrm{L}\right)$ & $142(1017-185)$ & $147(110-197 \rrbracket$ & $140(105-180)$ & $\begin{array}{l}P= \\
0.293\end{array}$ \\
\hline $\mathrm{CRP}(\mathrm{mg} / \mathrm{L})$ & $16.6(10.0-$ & $13.5(10.0-42.2)$ & $22.8(10.0-$ & $P=$ \\
\hline
\end{tabular}




\begin{tabular}{|c|c|c|c|c|}
\hline & $50.4)$ & & $56.33)$ & 0.086 \\
\hline Total cholesterol (mmol/L) & $\begin{array}{l}2.76(2.10- \\
3.49)\end{array}$ & $2.27(1.89-2.77)$ & $\begin{array}{l}3.26(2.49- \\
3.96)\end{array}$ & $\begin{array}{l}P= \\
0.000\end{array}$ \\
\hline $\mathrm{SBP}(\mathrm{mmHg})$ & $135(118-155)$ & $136(120-155)$ & $135(114-179)$ & $\begin{array}{l}P= \\
0.522\end{array}$ \\
\hline $\mathrm{DBP}(\mathrm{mmHg})$ & $78(65-89)$ & $80(66-89)$ & $76(62-88)$ & $\begin{array}{l}P= \\
0.108\end{array}$ \\
\hline HR (times/min) & $80 \pm 17$ & $78(66-89)$ & $76(62-88)$ & $\begin{array}{l}P= \\
0.278\end{array}$ \\
\hline CK-MB (U/L) & $12.0(7.7-23.0)$ & $10.7(6.4-14.0)$ & $16.8(9.0-30.3)$ & $\begin{array}{l}P= \\
0.000\end{array}$ \\
\hline $\begin{array}{l}\text { Hypersensitive troponin } \mathrm{T} \\
(\mathrm{ng} / \mathrm{mL})\end{array}$ & $\begin{array}{l}0.015(0.010- \\
0.042)\end{array}$ & $\begin{array}{l}0.012(0.010- \\
0.021)\end{array}$ & $\begin{array}{l}0.018(0.011- \\
0.102)\end{array}$ & $\begin{array}{l}P= \\
0.003\end{array}$ \\
\hline Ventilator-assisted time (d) & $2(1-6)$ & $2(1-4)$ & $3(1-8)$ & $\begin{array}{l}P= \\
0.014\end{array}$ \\
\hline Length of stay in ICU (d) & $6(4-11)$ & $6(4-9)$ & $7(4-12)$ & $\begin{array}{l}P= \\
0.113\end{array}$ \\
\hline The length of time (d) & $18(14-22)$ & $18(14-21)$ & $18(13-22)$ & $\begin{array}{l}P= \\
0.530\end{array}$ \\
\hline Infection, $n(\%)$ & $84(29)$ & $29(21)$ & $55(36)$ & $\begin{array}{l}P= \\
0.008\end{array}$ \\
\hline $\begin{array}{l}\text { Ventricular arrhythmias, } n \\
(\%)\end{array}$ & $15(5)$ & $4(3)$ & $11(7)$ & $\begin{array}{l}P= \\
0.110\end{array}$ \\
\hline Atrial arrhythmia, $n(\%)$ & $10(3)$ & $4(3)$ & $6(4)$ & $\begin{array}{l}P= \\
0.665\end{array}$ \\
\hline $\begin{array}{l}\text { Secondary tracheal } \\
\text { intubation, } n(\%)\end{array}$ & $47(16 \rrbracket$ & $17(13)$ & $30(19)$ & $\begin{array}{l}P= \\
0.113\end{array}$ \\
\hline $\begin{array}{l}\text { Secondary surgical } \\
\text { hemostasis, } n(\%)\end{array}$ & $11(4 \rrbracket$ & $3(2 \rrbracket$ & $8(5)$ & $\begin{array}{l}P= \\
0.188\end{array}$ \\
\hline Cerebral infarction, $n(\%)$ & $18(10)$ & $5(4)$ & $13(8)$ & $\begin{array}{l}P= \\
0.096\end{array}$ \\
\hline Cerebral hemorrhage, $n(\%)$ & $10(3)$ & $2(1)$ & $8(5)$ & $\begin{array}{l}P= \\
0.085\end{array}$ \\
\hline $\begin{array}{l}\text { Gastrointestinal dysfunction, } \\
n(\%)\end{array}$ & $53(18)$ & $14(10)$ & $39(25)$ & $\begin{array}{l}P= \\
0.001\end{array}$ \\
\hline Death, $n(\%)$ & $69(24)$ & $21(16)$ & $48(31)$ & $\begin{array}{l}P= \\
0.002\end{array}$ \\
\hline
\end{tabular}

To understand the combined value of UAR, age, and D-dimer in predicting deaths in patients with type A 


\begin{tabular}{|c|c|c|c|c|}
\hline Variable & All $(n=289)$ & $\begin{array}{l}\text { Survivor } \\
(n=220)\end{array}$ & $\begin{array}{l}\text { Non-survivor } \\
(n=69)\end{array}$ & $P$-value \\
\hline Age (years) & $52(44-59)$ & $51(43-57)$ & $58(50-67)$ & $\begin{array}{l}P \\
=0.000\end{array}$ \\
\hline Men, $n(\%)$ & $213(74)$ & $168(76)$ & $45(65)$ & $\begin{array}{l}P \\
=0.067\end{array}$ \\
\hline $\mathrm{BMI}\left(\mathrm{kg} / \mathrm{m}^{2}\right)$ & $\begin{array}{l}25.83(22.86- \\
28.15)\end{array}$ & $\begin{array}{l}25.51(22.86- \\
28.07)\end{array}$ & $\begin{array}{l}26.67(22.79- \\
28.44)\end{array}$ & $\begin{array}{l}P \\
=0.234\end{array}$ \\
\hline Smoking index (per year) & $0(0-400)$ & $0(0-400)$ & $0(0-400)$ & $\begin{array}{l}P \\
=0.727\end{array}$ \\
\hline Body mass index, $\left(\mathrm{kg} / \mathrm{m}^{2}\right)$ & $22(8)$ & $18(8)$ & $4(6)$ & $\begin{array}{l}P \\
=0.515\end{array}$ \\
\hline History of CHD (\%区 & $11(4)$ & $7(3)$ & $4(6)$ & $\begin{array}{l}P \\
=0.322\end{array}$ \\
\hline Hypertension, $n(\%)$ & $170(59)$ & $121(60)$ & $49(71)$ & $\begin{array}{l}P \\
=0.018\end{array}$ \\
\hline Diabetes, $n(\%)$ & $5(2)$ & $4(2)$ & $1(1)$ & $\begin{array}{l}P \\
=0.838\end{array}$ \\
\hline Euroscore $\rrbracket(\%)$ & $4.65(3.77-5.64)$ & $3.88(3.77-5.04)$ & $5.57(3.77-7.87)$ & $\begin{array}{l}P \\
=0.000\end{array}$ \\
\hline Uric acid ( $\mu \mathrm{mol} / \mathrm{L})$ & $421.29 \pm 88.02$ & $365.68 \pm 138.64$ & $382.62 \pm 119.83$ & $\begin{array}{l}P \\
=0.362\end{array}$ \\
\hline Albumin (g/L) & $35.7(33.0-38.6)$ & $36.2(33.6-38.9)$ & $34.2(31.5-36.8)$ & $\begin{array}{l}P \\
=0.000\end{array}$ \\
\hline Globulin (g/L) & $23.6(20.7-26.5)$ & $23.6(20.9-26.6)$ & $23.6(18.5-26.4)$ & $\begin{array}{l}P \\
=0.222\end{array}$ \\
\hline Total bilirubin ( $\mu \mathrm{mol} / \mathrm{L})$ & $18.0(14.4-26.9)$ & $\begin{array}{l}18.00(14.7- \\
27.2)\end{array}$ & $\begin{array}{l}17.40(13.8- \\
24.4)\end{array}$ & $\begin{array}{l}P \\
=0.290\end{array}$ \\
\hline UAR $(\mu \mathrm{mol} / \mathrm{g})$ & $\begin{array}{l}10.24(7.96- \\
12.54)\end{array}$ & $\begin{array}{l}9.65(7.60- \\
11.96)\end{array}$ & $\begin{array}{l}11.03(8.98- \\
13.08)\end{array}$ & $\begin{array}{l}P \\
=0.003\end{array}$ \\
\hline BUN (mmol/L) & $7.13(5.64-9.04)$ & $7.06(5.32-8.74)$ & $7.47(6.20-9.84)$ & $\begin{array}{l}P \\
=0.011\end{array}$ \\
\hline $\mathrm{Cr}(\mu \mathrm{mol} / \mathrm{L})$ & $78(58-105)$ & $73(54-99)$ & $91(68-122)$ & $\begin{array}{l}P \\
=0.000\end{array}$ \\
\hline $\begin{array}{l}\text { Creatinine clearance } \\
(\mathrm{mL} / \mathrm{min})\end{array}$ & $\begin{array}{l}99.4(69.9- \\
134.3)\end{array}$ & $\begin{array}{l}107.3(79.0- \\
147.2)\end{array}$ & $\begin{array}{l}71.8(55.5- \\
103.6)\end{array}$ & $\begin{array}{l}P \\
=0.000\end{array}$ \\
\hline $\operatorname{HGB}(\mathrm{g} / \mathrm{L})$ & $129(116-142)$ & $131(118-142)$ & $123(109-141)$ & $\begin{array}{l}P \\
=0.083\end{array}$ \\
\hline
\end{tabular}




\begin{tabular}{|c|c|c|c|c|}
\hline WBC $\left(\times 10^{9} / \mathrm{L}\right)$ & $\begin{array}{l}10.76(8.32- \\
14.43)\end{array}$ & $\begin{array}{l}10.65(8.28- \\
14.34)\end{array}$ & $\begin{array}{l}11.73(9.13- \\
14.95)\end{array}$ & $\begin{array}{l}P \\
=0.131\end{array}$ \\
\hline $\mathrm{RBC}\left(\times 10^{12} / \mathrm{L}\right)$ & $4.52 \pm 0.86$ & $4.21 \pm 0.63$ & $4.06 \pm 0.63$ & $\begin{array}{l}P \\
=0.039\end{array}$ \\
\hline $\operatorname{PLT}\left(\times 10^{9} / \mathrm{L}\right)$ & $142(107-185)$ & $154(113-190)$ & $118(86-155)$ & $\begin{array}{l}P \\
=0.000\end{array}$ \\
\hline CRP (mg/L) & $10.0(8.0-12.0)$ & $\begin{array}{l}14.9(10.0- \\
48.10)\end{array}$ & $23.7(10.0-73.0)$ & $\begin{array}{l}P \\
=0.091\end{array}$ \\
\hline Total cholesterol (mmol/L) & $3.44(2.89-4.05)$ & $3.50(2.96-4.06)$ & $3.23(2.53-3.95)$ & $\begin{array}{l}P \\
=0.065\end{array}$ \\
\hline SBP $(m m H g)$ & $135(118-155)$ & $138(120-158)$ & $124(110-148)$ & $\begin{array}{l}P \\
=0.010\end{array}$ \\
\hline $\mathrm{DBP}(\mathrm{mmHg})$ & $78(65-89)$ & $79(68-89)$ & $75(62-85)$ & $\begin{array}{l}P \\
=0.051\end{array}$ \\
\hline HR (times/min) & $80(68-90)$ & $80(68-90 \rrbracket$ & $80(67-70)$ & $\begin{array}{l}P \\
=0.634\end{array}$ \\
\hline CK-MB (U/L) & $12.0(7.7-23.0)$ & $12.0(7.0-20.0 \otimes$ & $18.0(9.2-32.5)$ & $\begin{array}{l}P \\
=0.002\end{array}$ \\
\hline $\begin{array}{l}\text { Hypersensitive troponin T } \\
\text { (ng/mL) }\end{array}$ & $\begin{array}{l}0.015(0.010- \\
0.042)\end{array}$ & $\begin{array}{l}0.013(0.010- \\
0.410 \rrbracket\end{array}$ & $\begin{array}{l}0.018(0.012- \\
0.044)\end{array}$ & $\begin{array}{l}P \\
=0.256\end{array}$ \\
\hline D-dimer (mg/L) & $\begin{array}{l}8.86(3.14- \\
20.33)\end{array}$ & $\begin{array}{l}7.20(2.67- \\
17.08 \rrbracket\end{array}$ & $\begin{array}{l}18.50(8.12- \\
30.93)\end{array}$ & $\begin{array}{l}P \\
=0.000\end{array}$ \\
\hline FDP & $\begin{array}{l}26.43(9.63- \\
61.78)\end{array}$ & $\begin{array}{l}22.70(7.52- \\
47.49)\end{array}$ & $\begin{array}{l}57.98(27.43- \\
107.47)\end{array}$ & $\begin{array}{l}P \\
=0.000\end{array}$ \\
\hline
\end{tabular}

AAD, deaths were evaluated using the ROC method (Table 5). The AUC of D-dimer combined with age was $0.731[95 \%(0.659,0.803)]$, and the sensitivity and specificity were $70.8 \%$ and $65.5 \%$, respectively $(P=$ 0.000). The AUC of UAR combined with both age and D-dimer was 0.751 [95\% $(0.681,0.821)]$, and the sensitivity and specificity were $76.8 \%$ and $68.2 \%$, respectively $(P=0.000)$. The AUC of UAR combined with D-dimer was 0.727 [95\% $(0.660,0.794)]$, and the sensitivity and specificity were $85.5 \%$ and $51.8 \%$, respectively $(P=0.000)$. The AUC of UAR combined with age was $0.714[95 \%(0.640,0.787)]$, and the sensitivity and specificity were $59.4 \%$ and $75.9 \%$, respectively $(P=0.000)$. Thus, the combination of UAR and D-dimer was best for predicting deaths (Figure 2 ). 


\begin{tabular}{|c|c|c|c|c|c|c|}
\hline \multirow[t]{2}{*}{ Variable } & \multicolumn{3}{|c|}{ Univariate Analysis } & \multicolumn{3}{|c|}{ Multivariate Analysis } \\
\hline & $\mathrm{HR}$ & $95 \% \mathrm{Cl}$ & P Value & HR & $95 \% \mathrm{Cl}$ & $P$-value \\
\hline UAR $₫ \mu \mathrm{mol} / \mathrm{g} \rrbracket$ & 2.4000 & $1.462-3.941$ & 0.001 & 1.904 & $1.097-3.305$ & 0.022 \\
\hline Age (years) & 2.870 & $1.784-4.618$ & 0.000 & 2.216 & $1.287-3.815$ & 0.004 \\
\hline Hypertension (\%) & 1.707 & $1.022-2.851$ & 0.041 & 1.825 & $1.077-3.094$ & 0.025 \\
\hline Albumin $(g / L)$ & 2.192 & $1.003-4.789$ & 0.049 & 1.660 & $0.734-3.754$ & 0.224 \\
\hline $\mathrm{CRP}(\mathrm{mg} / \mathrm{L})$ & 1.723 & $1.059-2.805$ & 0.028 & 1.663 & $0.998-2.773$ & 0.051 \\
\hline Creatinine $(\mu \mathrm{mol} / \mathrm{L})$ & 2.198 & $1.316-3.672$ & 0.003 & 1.602 & $0.905-2.838$ & 0.106 \\
\hline $\operatorname{RBC}\left(\times 10^{12} / \mathrm{L}\right)$ & 1.664 & $1.022-2.708$ & 0.041 & 1.364 & $0.772-2.409$ & 0.285 \\
\hline Platelets $\left(\times 10^{9} / \mathrm{L}\right)$ & 1.824 & $1.137-2.925$ & 0.013 & 1.096 & $0.633-1.898$ & 0.744 \\
\hline CK-MB (U/L) & 1.717 & $1.028-2.868$ & 0.039 & 1.035 & $0.587-1.824$ & 0.906 \\
\hline D-dimer (mg/L) & 2.780 & $1.684-4.590$ & 0.000 & 1.991 & $1.116-3.554$ & 0.020 \\
\hline $\mathrm{FDP}(\mathrm{mg} / \mathrm{L})$ & 3.543 & $2.083-6.028$ & 0.000 & 1.811 & $0.934-3.512$ & 0.079 \\
\hline
\end{tabular}

Table 3. Univariate and multivariate predictor analysis results for all-cause death

\begin{tabular}{|lcllllll|}
\hline Variables & AUC & $\begin{array}{l}\text { Cut-off } \\
\text { value }\end{array}$ & SE & $95 \% \mathrm{Cl}$ & Sensitivity & Specificity & $P$-value \\
\hline UAR & 0.618 & $>9.875$ & 0.038 & $\begin{array}{l}0.544- \\
0.693\end{array}$ & 0.696 & 0.518 & 0.003 \\
\hline Age (years) & 0.685 & $>56$ & 0.039 & $\begin{array}{l}0.609- \\
0.761\end{array}$ & 0.565 & 0.741 & 0.000 \\
\hline Hypertension (\%) & 0.573 & $>0.5$ & 0.039 & $\begin{array}{l}0.497- \\
0.649\end{array}$ & 0.696 & 0.450 & 0.068 \\
\hline Albumin (g/L) & 0.349 & $<36.45$ & 0.039 & $\begin{array}{l}0.274- \\
0.425\end{array}$ & 0.261 & 0.518 & 0.000 \\
\hline D-dimer (mg/L) & 0.701 & $>9.19$ & 0.035 & $\begin{array}{l}0.632- \\
0.770\end{array}$ & 0.725 & 0.600 & 0.000 \\
\hline $\begin{array}{l}\text { Uric acid } \\
\text { ( } \mathrm{mmol} / \mathrm{L})\end{array}$ & 0.544 & $>305$ & 0.038 & $\begin{array}{l}0.470- \\
0.619\end{array}$ & 0.783 & 0.345 & 0.265 \\
\hline
\end{tabular}

Table 4. Predictive value of UAR, D-dimer, age, hypertension, albumin, and uric acid for mortality in patients with type A AAD 


\begin{tabular}{|lllllll|}
\hline Variables & AUC & SE & $95 \% \mathrm{Cl}$ & Sensitivity & Specificity & $P$-value \\
\hline UAR + age & 0.714 & 0.038 & $0.640-0.787$ & 0.594 & 0.759 & 0.000 \\
\hline UAR+D-dimer & 0.727 & 0.034 & $0.660-0.794$ & 0.855 & 0.518 & 0.000 \\
\hline UAR + age + D-dimer & 0.751 & 0.036 & $0.681-0.821$ & 0.768 & 0.682 & 0.000 \\
\hline D-dimer+ age & 0.731 & 0.037 & $0.659-0.803$ & 0.708 & 0.655 & 0.000 \\
\hline
\end{tabular}

Predictive values of different combinations of indicators (D-dimer + age, UAR + age + D-dimer, UAR + Ddimer, and UAR + age) for mortality in patients with type A AAD.

\section{Discussion}

Type A AAD is one of the most common and critical diseases in cardiovascular surgery. The early identification of risk factors in patients with type A AAD may help reduce the risk of death in these patients. Old age, a history of hypertension, cardiac tamponade, shock, coronary tear, acute renal failure, acute liver failure, stroke, mesenteric ischemia, and other risk factors have been considered as independent predictors of death in patients with type A AAD [15]; however, these risk factors cannot meet the needs of early diagnosis in clinical practice. In this study, we found that age, history of hypertension, preoperative UAR, serum albumin, D-dimer, and FDP in patients with Type A AAD were associated with the risk of one-year mortality in type A AAD patients, and preoperative UAR, D-dimer, and age were identified as independent risk factors. These findings agree with other studies that identified D-dimer and age as independent predictors of death in type A AAD patients[16,17]. This is the first study to evaluate the predictive effect of UAR for mortality in type A AAD. Thus, UAR can be considered as a new independent predictor of death in type A AAD.

SA is likely protection factors to be the type A AAD.when type A AAD occurs, a large number of inflammatory cytokines release, increase SA decomposition and led to lower SA. lower SA caused oedema after large amounts of fluid leakage and reduced the blood volume. ,AKI, pulmonary edema, cerebral edema and other serious complications can further affect the postoperative outcome of patients with Type A AAD. Low SA reduces the ability of scavenging oxygen free radicals and inhibiting inflammatory response, and endothelial cells are damaged, and the elasticity of aortic vascular wall decreases. Ultimately affecting the outcome of Type A AAD $[18,19]$.

Uric acid, which is the final product of purine metabolism, is produced by a xanthine oxidoreductasecatalyzed oxidation reaction. The concentration of uric acid in serum is related to the total antioxidant capacity and aortic dilation [20] along with pathological processes such as endothelial dysfunction, oxidative stress, systemic inflammation, and renin-angiotensin system activation [21-22]. These pathologic processes further lead to endothelial dysfunction, vascular smooth muscle cell proliferation, and increased arterial stiffness. Uric acid can also increase the serum contents of IL-1 $\beta$ and nucleotide binding oligomerization domain-like receptor 3 along with blood pressure variability. Hyperuricemia can participate in aortic vascular endothelial injury through an oxidative stress pathway, resulting in increased 


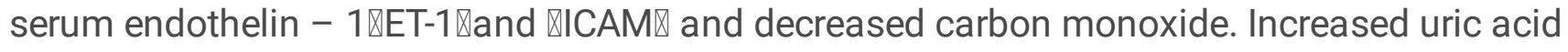
concentration led to a decrease in $\triangle \mathrm{ENOS} \otimes$ expression, increased ET-1 and ICAM expressions, and a significant increase in the apoptosis rate of aortic cells[23]. Zhang reported that preoperative uric acid was an independent risk factor for postoperative in-hospital death in type A AAD [24]. However, Zhang's study considered few risk factors for type A AAD, did not exclude confounding factors, involved only a small number of patients, and did not include long-term follow-up. In clinical practice, many factors affect the production and excretion of SA and uric acid [25].

Çakmak reported that UAR was an independent predictor of the severity of coronary artery disease but did not assess type A AAD specifically [14]. In this study, we focused on patients diagnosed with type A AAD and treated with surgery. Follow-up over one year after surgery revealed that UAR can better predict the mortality than the serum albumin or uric acid content. We also assessed the values of age and D-dimer for predicting death in type A AAD patients since these indicators have been identified as important risk factors in previous studies. We found that the combination of UAR, age, and D-dimer had better predictive value for death than separate of UAR, age, and D-dimer. This may be due to the exposure of subcutaneous tissue caused by intima tear during aortic dissection, resulting in the release of tissue factors that increase the consumption and metabolism of albumin [26]. Inflammation plays an important role in the entire pathophysiological continuum of type $A A A D$, and the severity of inflammation is related to type A AAD rupture and prognosis [27]. SA is an important inhibitor of platelet activation and aggregation, and reduced SA levels may be associated with an increased risk of thrombotic events associated with increased PLT activation and aggregation [28]. Low serum albumin concentration leads to reduced antioxidant activity, and oxidative stress is important in the pathology of type A AAD [29]. Renal insufficiency is a part of complications of type $A A A D$, and uric acid is one of the indexes of early renal damage. In addition, uric acid can damage endothelial function and lead to oxidative stress, systemic inflammation, renin-angiotensin system activation, and increased apoptosis rate. In our study, uric acid and SA were combined to exclude confounding factors. Serum albumin or uric acid alone has poor specificity in predicting death, while albumin combined with uric acid can predict the outcome of type A AAD. However, the combination of UAR, age, and D-dimer was a better predictor of death.

\section{Conclusions}

In patients with type A AAD, UAR may be considered as an independent risk factor for long-term mortality. The predictive value of UAR was superior to that of albumin and uric acid. Among the tested indicators and their combinations, the combination of UAR, age, and D-dimer was the best predictor of death. UAR may improve the accuracy of clinical prognosis and shows promise for clinical application because its determination is low cost, quick, and simple. Therefore, UAR shows value for evaluating the prognosis in type A AAD patients to guide future clinical research and treatment strategy.

\section{Abbreviations}

AAD acute aortic dissection 
UAR uric acid-to-albumin Ratio

COX proportional hazards model

ROC receiver operating characteristic

AUC receiver operating characteristic curve

SA serum albumin

\section{Limitations}

First, this study was a single-center observational study. Although follow-up was conducted, no further review was carried out. Therefore, a prospective, large-scale, and multi-center study is needed to confirm our conclusions. Second, this study was failed to consider only preoperative UAR, future research is to consider more center more number of patients, prospective study, calculation UAR with dynamic monitoring, to evaluate UAR affecting type A AAD patient outcome in different time points.

\section{Acknowledgements}

The authors thank AiMi Academic Services (www.aimieditor.com) for the English language editing and review services.This study was supported by Key R \& D project of Shaanxi Province(No. 2018ZDCXL-SF26-1)

\section{Authors' contributions}

X.W.completed the study design, data analysis, manuscript preparation, and manuscript editing; C.D. and FW.G. conducted the literature review and clinical study; Q.M and M.L participated in the experimental studies; YB.X .and L.Z.completed the data acquisition; X.W. and H.G. and XP.Z.contributed to the statistical analysis, the study concepts, definition of intellectual content, and manuscript review. All authors read and approved the final manuscript.

\section{Data availability}

All data generated or analyzed during this study are included in this published article.

\section{Competing interests}

The authors declare that they have no competing interest.

\section{Author details}

1 Department of Cardiovascular Surgery, First Affiliated Hospital of Xi'an

Jiaotong University, No. 277 Yanta West Road, Xi'an 710061, People's Republic 
of China. 2 Department of Emergency Internal Medicine, Shaanxi Provincial

People's Hospital, 256 Youyi West Road, Xi'an 710068, People's Republic

\section{Funding}

Key R \& D project of Shaanxi Province(No. 2018ZDCXL-SF-26-1).

\section{Additional information}

Correspondence and requests for materials should be addressed to X.W.

\section{References}

1. Pape LA,et al. Aortic Diameter $\geq 5.5 \mathrm{~cm}$ Is Not a Good Predictor of Type A Aortic Dissection: Observations From the International Registry of Acute Aortic Dissection (IRAD). Circulation 116,1120-1127.(2007).

2. Trimarchi S, Nienaber CA, Rampoldi V,et al. Contemporary results of surgery in acute type A aortic dissection: the International Registry of Acute Aortic Dissection experience. J.Thorac Cardiovasc Surg. 129,112-122(2005).

3. Tolis, Jr and Sundt III. Contemporary insights into the management of type A aortic dissection. J.Expert Review of Cardiovascular Therapy 14,1189-1196(2016).

4. Zhang WJ, Frei B. Albumin selectively inhibits TNF alpha-induced expression of vascular cell adhesion molecule-1 in human aortic endothelial cells. Cardiovasc Res.55,820-829(2002).

5. Ronit A,et al. Plasma albumin and incident cardiovascular disease: results from the CGPS and an updated meta-analysis. Arterioscler Thromb Vasc Biol.40,473-82 (2020).

6. Arques S. Human serum albumin in cardiovascular diseases. Eur J Intern Med .52,8-12(2018).

7. Liao LZ,et al. Serum albumin and atrial fibrillation: insights from epidemiological and Mendelian randomization studies. Eur J Epidemiol 35,113-22(2020).

8. Nagai T,et al. JASPER Investigators. Clinical characteristics, management and outcomes of japanese patients hospitalized for heart failure with preserved ejection fraction -a report from the japanese heart failure syndrome with preserved ejection fraction (JASPER) registry. Circ J .82,1534-45(2018).

9. Maruhashi T;Hisatome I;Kihara Y;Higashi Y. Hyperuricemia and endothelial function: From molecular background to clinical perspectives. J.Atherosclerosis 278,226-231(2018).

10. Papezikova I;Pekarova M;Lojek A;Kubala L. The effect of uric acid on homocysteine-induced endothelial dysfunction in bovine aortic endothelial cells.J. Neuro Endocrinol Lett.30,112-115 (2009).

11. Cai J, et al. Serum uric acid could be served as an independent marker for increased risk and severity of ascending aortic dilatation in Behçet's disease patients. J .Clin Lab Anal.33,e22637(2019). 
12. VIRDIS A,et al.Identification of the uric acid thresholds predicting an increased total and cardiovascular mortality over 20 years.J.Hypertension 75,302-308(2020).

13. KLEBER ME,et al.Uric acid and cardiovascular events: a mendelian randomization study.J.Am Soc Nephrol.26,2831-2838(2015).

14. Çakmak EÖ, et al. Uric Acid-to-Albumin Ratio: A Novel Marker for the Extent of Coronary Artery Disease in Patients with Non-ST-Elevated Myocardial Infarction. J.Pulse (Basel) 8,99-107(2021).

15. Evangelista, A. et al. Insights from the international registry of acute aortic dissection: A 20-year experience of collaborative clinicalresearch. Circulation 137, 1846-1860 (2018)

16. Flanagan, L., Bancrof, R. \& Rittoo, D. The value of d-dimer in the diagnosis of acute aortic dissection. Int. J. Cardiol. 118, e70-71 (2007).

17. Marill, K. A. Serum d-dimer is a sensitive test for the detection of acute aortic dissection: A pooled meta-analysis. J. Emerg. Med. 34, 367-376 (2008)

18. Keskin HA, et al. Prognostic nutritional index predicts in-hospital mortality in patients with acute Stanford type A aortic dissection.J.Perfusion 36,710-716(2021).

19. Zhao X, Bie M. Predictors for the development of preoperative oxygenation impairment in acute aortic dissection in hypertensive patients. BMC Cardiovasc Disord 20,365(2020).

20. Esen AM, et al. Uric acid as a marker of oxidative stress in dilatation of the ascending aorta. Am J Hypertens. 24,149-54(2011).

21. Ruggiero C, et al. Uric acid and inflammatory markers. Eur Heart J. 27,1174-81(2006)

22. Mehta T, et al. Association of Uric Acid with Vascular Stiffness in the Framingham heart study. Am J Hypertens. 28,877-83(2015).

23. BALAKUMAR P, SHARMA R, SINGH M. Benfotiamine attenuates nicotine and uric acid-induced vascular endothelial dysfunction in the rat. J.Pharmacol Res. 58, 356-363(2008).

24. Yiran Zhang,et al. Preoperative uric acid predicts in-hospital death in patients with acute type a aortic dissection[J]. Journal of Cardiothoracic Surgery. 15,112-122(2020).

25. Arques S. Human serum albumin in cardiovascular diseases. Eur J Intern Med.52,8-12(2018).

26. Artigas A, et al. Role of albumin in diseases associated with severe systemic inflflammation: pathophysiologic and clinical evidence in sepsis and in decompensated cirrhosis. J.Crit Care.33,6270(2016).

27. Cifani N, et al. Stanford-A acute aortic dissection, inflflammation, andmetalloproteinases: a review. Ann Med.47,441-6(2015).

28. $\mathrm{Li} \mathrm{HH}$, et al. Cytoprotective effects of albumin, nitrosated or reduced, in cultured rat pulmonary vascular cells. Am J Physiol Lung Cell Mol Physiol.300,L526-33(2011).

29. Abe N, et al. A 2-year follow-up of oxidative stress levels in patients with ST-segment elevation myocardial infarction: a subanalysis of the ALPS-AMI study. Angiology 66,271-277(2015).

\section{Figures}




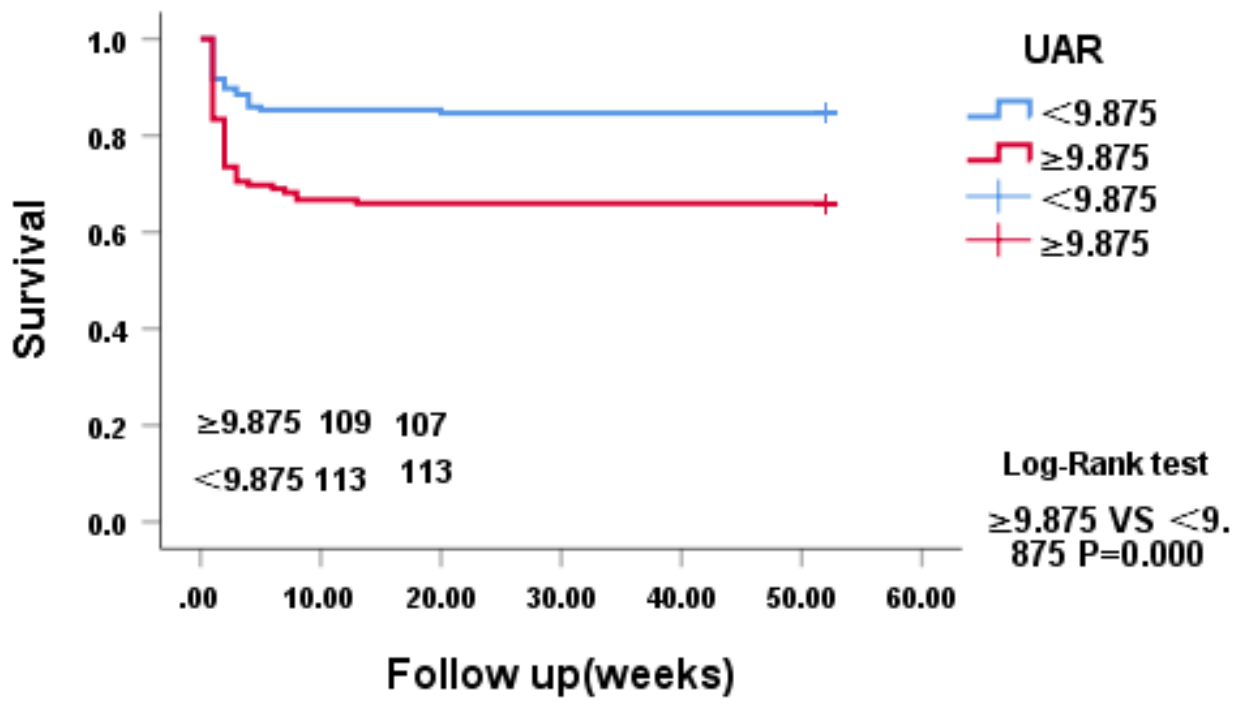

Figure 1

Cumulative survival in each group.
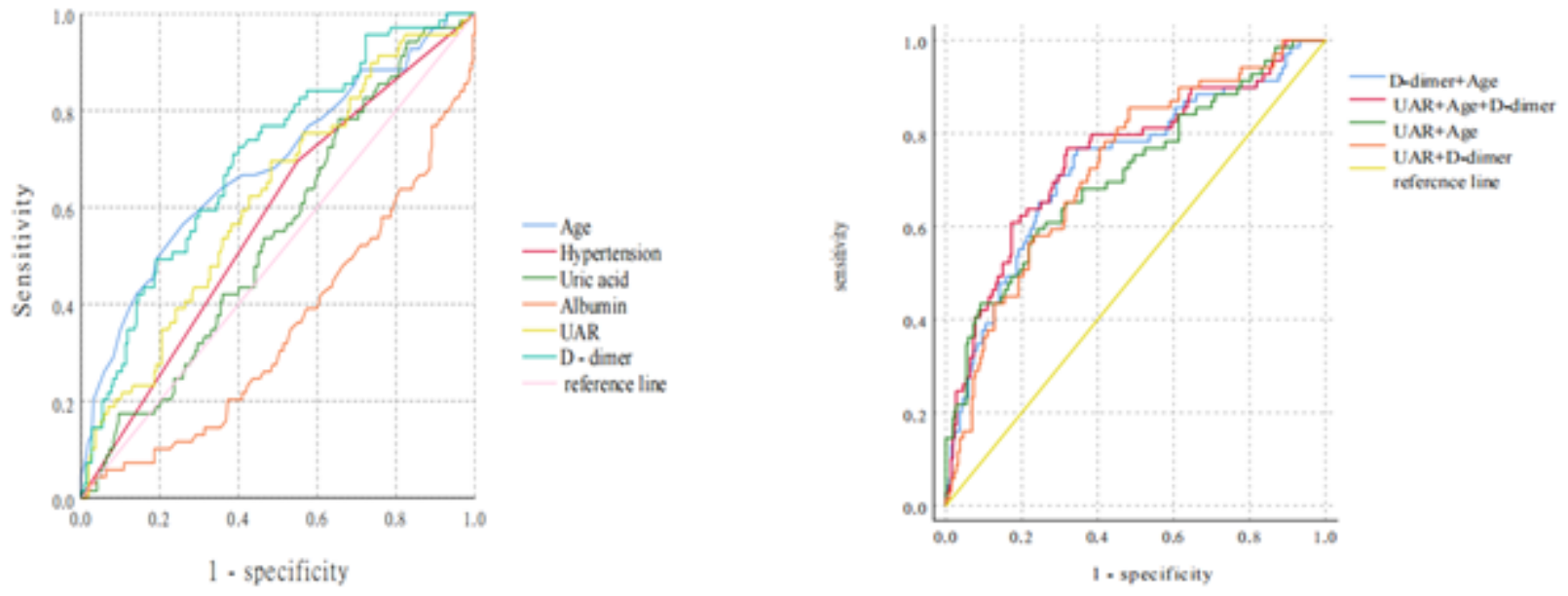

Figure 2

ROC curves of single indicators for predicting death in patients with type A AAD. 

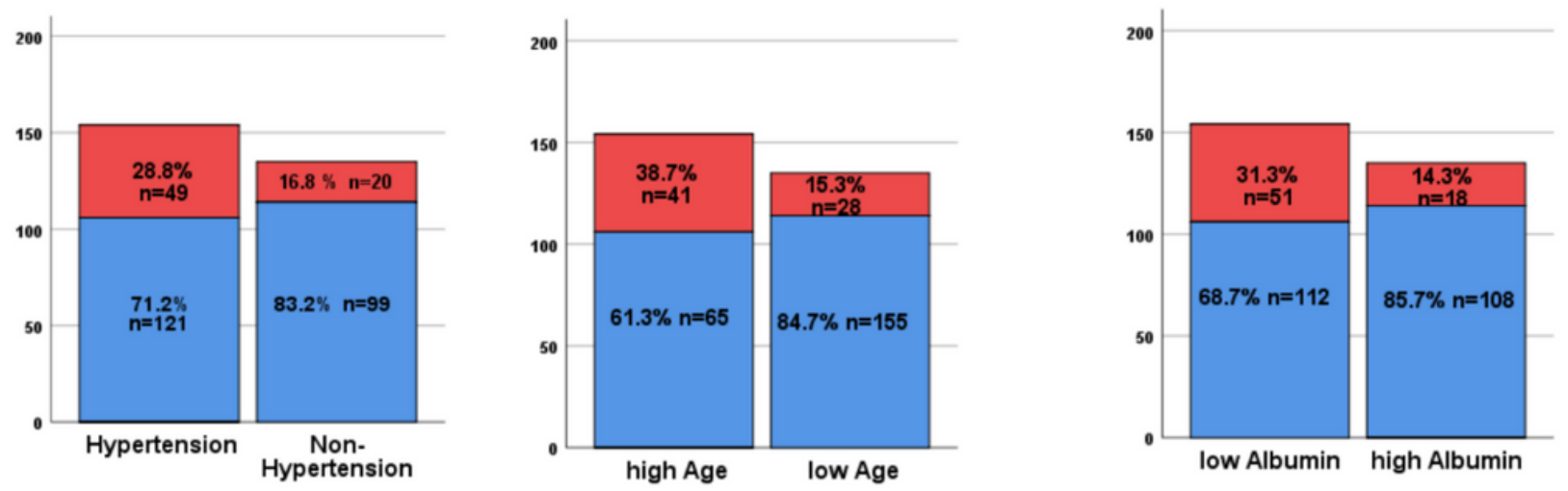

Non-survivor

Survivor
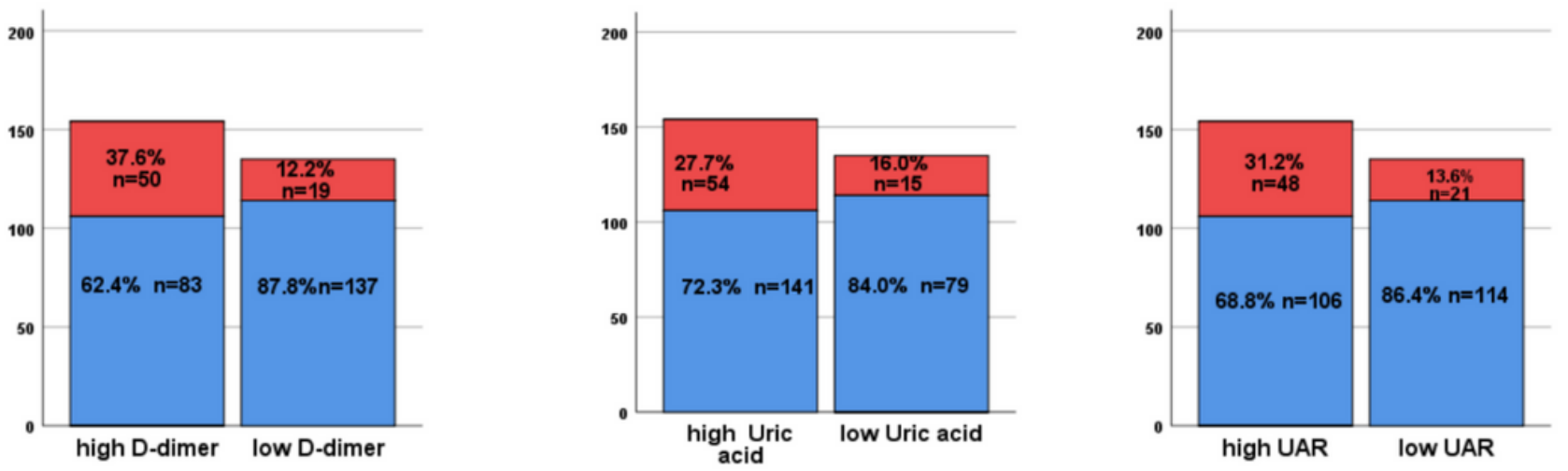

\section{Figure 3}

Mortality rates in patients with type A AAD in different groups based on UAR, D-dimer, age, hypertension, albumin, and uric acid. 